\title{
Michael Struwe
}

A counterexample in regularity theory for parabolic systems

Czechoslovak Mathematical Journal, Vol. 34 (1984), No. 2, 183-188

Persistent URL: http://dml.cz/dmlcz/101942

\section{Terms of use:}

(C) Institute of Mathematics AS CR, 1984

Institute of Mathematics of the Czech Academy of Sciences provides access to digitized documents strictly for personal use. Each copy of any part of this document must contain these Terms of use.

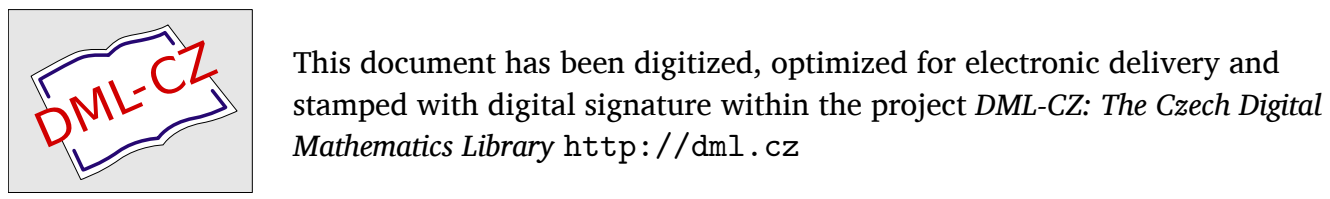




\title{
A COUNTEREXAMPLE IN REGULARITY THEORY FOR PARABOLIC SYSTEMS
}

\author{
Michael Struwe ${ }^{1}$ ), Bonn
}

(Received June 18, 1982)

\section{INTRODUCTION}

Let $\Omega$ be a smoothly bounded open set in $\mathbb{R}^{n}, Q=\Omega \times[0, \infty[$ and let $L$ be a uniformly elliptic operator given by

with coefficients

$$
L u=-\partial_{\alpha} a^{\alpha \beta} \partial_{\beta} u
$$

satisfying for all $\xi \in \mathbb{R}^{n}$

$$
a^{\alpha \beta} \in L^{\infty}(Q)
$$

$$
a^{\alpha \beta} \xi_{\alpha} \xi_{\beta} \geqq \lambda|\xi|^{2}
$$

with a uniform constant $\lambda>0$. Moreover, let $f: Q \times \mathbb{R}^{N+n N} \rightarrow \mathbb{R}^{N}$ be a Carathéodory function and assume that

$$
|f(x, t, u, p)| \leqq a|p|^{2}+b
$$

for all $(x, t, u, p) \in Q \times \mathbb{R}^{N+n N}$ with constants $a, b \in \mathbb{R}$.

Consider the Cauchy-Dirichlet problem for the quasilinear diagonal parabolic system

$$
\begin{gathered}
\partial_{t} u^{i}+L u^{i}=f^{i}(x, t, u, \nabla u), \quad 1 \leqq i \leqq N \\
u \mid\left(\partial \Omega \times\left[0, \infty[) \cup(\Omega \times\{0\})=u_{0}\right.\right.
\end{gathered}
$$

for smooth boundary and initial data $u_{0}$ and assume that $u \in L^{\infty}\left(Q ; \mathbb{R}^{N}\right) \cap$ $\cap L_{\text {loc }}^{2}\left(\left[0, \infty\left[; H^{1,2}\left(\Omega ; \mathbb{R}^{N}\right)\right)\right.\right.$ is a weak solution to $(3)$ in the sense that

$$
u(\cdot, t)-u_{0}(\cdot, t) \in H_{0}^{1,2}\left(\Omega ; \mathbb{R}^{N}\right) \text { a.e. in }[0, \infty[
$$

and that the relation

(4)

$$
\int_{Q}\left[-u^{i} \partial_{t} \varphi^{i}+a^{\alpha \beta} \partial_{\beta} u^{i} \partial_{\alpha} \varphi^{i}\right] \mathrm{d} x \mathrm{~d} t=\int_{Q} f^{i}(x, t, u, \nabla u) \varphi^{i} \mathrm{~d} x \mathrm{~d} t+\int_{\Omega \times\{0\}} u_{0} \varphi \mathrm{d} x
$$

$\left.{ }^{1}\right)$ This research was supported by the Sonderforschungsbereich 72 of the Deutsche Forschungsgemeinschaft. 
is satisfied for all $\varphi \in C^{\infty}(Q)$ vanishing in a neighborhood of $\partial \Omega \times[0, \infty[$ and also vanishing for large $t$.

In [7], [2] it was shown that such a weak solution $u$ is Hölder continuous (and hence regular) in the interior of $Q$ if the ellipticity constant $\lambda$ in (1), the growth coefficient $a$ in (2) and $M=\|u\|_{\infty}$ are related by the condition

$$
a M<\lambda .
$$

Moreover, it was noted that condition (5) is best possible. Indeed, on $\Omega=$ $=\left\{x \in \mathbb{R}^{3}|| x \mid<1\right\}$, the function $h \in H^{1,2} \cap L^{\infty}\left(\Omega ; \mathbb{R}^{3}\right)$ given by

$$
h(x)=x|x|^{-1}
$$

weakly solves the diagonal elliptic system

$$
-\Delta h=h|\nabla h|^{2}
$$

but is discontinuous at $x=0$. Since we may interpret $h$ as a stationary weak solution of a parabolic system (3) with $a=M=\lambda=1$ condition (5) in general cannot be improved.

However, the above example due to Hildebrandt-Widman [4] and other counterexamples to regularity in the case of elliptic systems given by Frehse [1] and Heinz [3] leave open the question of whether the parabolic "flow" preserves regularity properties of the initial and boundary data as the solution evolves in time, even without any restriction on the size of the ratio $a M / \lambda$. In fact, it was conjectured by Sampson [6] that parabolic systems arising from harmonic mappings between Riemannian manifolds act conservative in this sense, and it is suspected that the regularity properties of the initial data will even be conserved if the solution is only bounded locally with respect to $t$.

However, the example below shows that in general the answer will be negative and that in general solutions of systems (3) may lose the regularity properties of the initial data in finite time if $a M / \lambda$ is too large. (We suspect that this will be true if $a M / \lambda>1$, already, leaving open the limiting case $a M=\lambda$.) Thus, the special (variational) structure of the operator must be held responsible for the phenomena occuring in the case of harmonic mappings.

Again, one should ask: Do parabolic systems of the kind (3) preserve regularity if they are of variational type? A positive response to this question should be of great interest for various applications in differential geometry.

Example. Let $\Omega=\left\{x \in \mathbb{R}^{3}|| x \mid<1\right\}$ be the unit ball in $\mathbb{R}^{3}, Q=\Omega \times[0, \infty[$. Let $\Gamma$ be the fundamental solution of the (backward) heat operator with singularity at $(0,1) \in Q$, i.e.

$$
\Gamma(x, t)=\frac{1}{(4 \pi|1-t|)^{3 / 2}} \exp \left(-\frac{x^{2}}{4|1-t|}\right)
$$

if $t<1, \Gamma(x, t)=0$, otherwise. 
By classical results we have the following representation formula for the solution $v$ of the initial value problem

$$
\begin{gathered}
\left.\left.-\partial_{t} v-\Delta v=0 \text { in } \mathbb{R}^{3} \times\right]-\infty, 1\right] \\
v(\cdot, 1)=v_{1}:
\end{gathered}
$$

For any $v_{1} \in L^{\infty}\left(\mathbb{R}^{3} ; \mathbb{P}\right)$ the unique solution $v$ of $(9)$ is given by

$$
v(x, t)=\int_{\mathbb{R}^{3}} \Gamma(x-y, t) v_{1}(y) \mathrm{d} y=:\left(\Gamma(\cdot, t) * v_{1}\right)(x) .
$$

Moreover, $v$ is smooth for $t<1$ and hence satisfies (9) in the pointwise classical sense (cp. [5, p. 262]). Also the initial data $v_{1}$ are assumed continuously in $L_{\text {loc }}^{2}\left(\mathbb{R}^{3} ; \mathbb{R}\right)$ as may easily be verified using again the estimates in $[5$, p. $262 \mathrm{f}$. $]$.

Now, define for $t \in \mathbb{R}, x \in \Omega$

$$
\begin{aligned}
& u(x, t)= \begin{cases}(2 h * \Gamma(\cdot, t))(x), & t<1 \\
2 h(\mathrm{x}) & t \geqq 1\end{cases} \\
& v(x, t)= \begin{cases}(2 h * \Gamma(\cdot, t))(x), & t<1 \\
2 h(x), & t=1 \\
(2 h * \Gamma(\cdot, 2-t))(x), & t>1\end{cases}
\end{aligned}
$$

where $h \in L^{\infty} \cap H_{\text {loc }}^{1,2}\left(\mathbb{R}^{3} ; \mathbb{R}^{3}\right)$ is given by (6). Also let

$$
L=-\alpha \Delta, \quad M=-\beta \Delta
$$

where $\alpha, \beta \in L^{\infty}(Q)$ are given by $\alpha \equiv \beta \equiv 3$ if $t<1, \alpha \equiv 4$ if $t \geqq 1, \beta \equiv 4$ if $t=1$, $\beta \equiv 5$ if $t>1$. Clearly, both $L$ and $M$ satisfy condition (1) with $\lambda=1$.

\section{Lemma 1.}

$$
u, v \in L^{\infty}\left(Q ; \mathbb{R}^{3}\right) \cap L_{\mathrm{loc}}^{2}\left(\left[0, \infty\left[; H^{1,2}\left(\Omega ; \mathbb{R}^{3}\right)\right) .\right.\right.
$$

Proof. Using the fact that $\Gamma \geqq 0$ and $\int \Gamma(x, t) \mathrm{d} x=1$ for $t<1$ the first assertion is a simple consequence of the convolution estimate

for all $\varphi \in L^{\infty}, \psi \in L^{1}$.

$$
\|\varphi * \psi\|_{\infty} \leqq\|\varphi\|_{\infty}\|\psi\|_{1}
$$

To verify the second part of the claim we show that $\|\nabla u(\cdot, t)\|_{2}$ is even bounded, uniformly in $t<1$. Indeed, using the smoothness of $\Gamma$ and the definition of weak derivative for $t<1$ we may write

$$
\nabla u=(2 h * \nabla \Gamma(\cdot, t))=(2 \nabla h * \Gamma(\cdot, t)) .
$$

Thus, by standard estimates and Fubini's theorem

$$
\int_{\Omega}|\nabla u(\cdot, t)|^{2} \mathrm{~d} x \leqq c \int_{\Omega} \int_{\mathbb{R}^{3}}|\nabla h(x-y)|^{2} \Gamma(y, t) \mathrm{d} x \mathrm{~d} y \leqq c \int_{\Omega}|\nabla h(x)|^{2} \mathrm{~d} x .
$$


Here, we also have used that $|\nabla h(x)|^{2}=2|x|^{-2}$, whence the integral of $|\nabla h|^{2}$ over any unit ball is dominated by that taken over $\Omega$.

Next, observe that $u$ weakly solves the parabolic system (cp. (9), (10)) qed.

$$
\begin{gathered}
\partial_{t} u+L u=\left(\partial_{t}+\Delta\right)(2 h * \Gamma(\cdot, t))-4 \Delta(2 h * \Gamma(\cdot, t))= \\
=(-4 \Delta(2 h) * \Gamma(\cdot, t))=\left(2 h|\nabla(2 h)|^{2} * \Gamma(\cdot, t)\right), \text { if } t<1 \\
\partial_{t} u+L u=u|\nabla u|^{2}, \quad \text { if } t>1
\end{gathered}
$$

hence a system of the type (3) if we formally define $f$ via "Tomi's trick":

$$
f(x, t, u, p)= \begin{cases}\frac{8\left(h|\nabla h|^{2} * \Gamma(\cdot, t)\right)(x)}{|\nabla u(x, t)|^{2}} p^{2}, & t<1 \\ 2 h(x) p^{2}, & t \geqq 1 .\end{cases}
$$

(Note that, again, we have used the fact that $\Gamma$ is smooth and decays rapidly at infinity for $t<1$ in order to differentiate under the integral sign and shift the differential to $h$. Also we have used (7).)

Similarly, $v$ is a solution of

$$
\begin{gathered}
\partial_{t} v+M v=f(x, t, v, \nabla v), \quad \text { if } t<1 \\
\partial_{t} v+M v=\left(\partial_{t}-\Delta\right)(2 h * \Gamma(\cdot, 2-t))-4 \Delta v= \\
=-f(x, 2-t, v, \nabla v), \quad \text { if } t>1 .
\end{gathered}
$$

To make the above calculations precise simply note

Lemma 2. The function $f$ is well defined on $\Omega \times]-\infty, 1\left[\times \mathbb{R}^{3} \times \mathbb{R}^{9}\right.$ and measurable in $(x, t) \in \Omega \times]-\infty, 1\left[\right.$ and continuous in $(u, p) \in \mathbb{R}^{3} \times \mathbb{R}^{9}$. Moreover, there exists a constant $a \in \mathbb{R}$ such that

$$
|f(x, t, u, p)| \leqq a|p|^{2} .
$$

Proof. Clearly, it suffices to show that there exists $a \in \mathbb{R}$ such that

$$
\left|2 h(x)\left(|\nabla(2 h)|^{2} * \Gamma(\cdot, t)\right)(x)\right| \leqq a|\nabla(2 h * \Gamma(\cdot, t))(x)|^{2} .
$$

Denoting absolute constants by $c$ and using the identity (with $\delta_{i j}=1$ if $i=j$, $\delta_{i j}=0$ if $i \neq j$ )

we have for $t<1$

$$
|\nabla h(x)|^{2}=\frac{2}{|x|^{2}}, \quad \nabla_{i} h^{j}(x)=\frac{\delta_{i j}|x|^{2}-x_{i} x_{j}}{|x|^{3}},
$$

$$
\begin{gathered}
\left|\left(h|\nabla h|^{2} * \Gamma(\cdot, t)\right)(x)\right|= \\
=\left|\int_{\mathbb{R}^{3}} \frac{2(x-y)}{|x-y|^{3}} \frac{1}{(4 \pi|1-t|)^{3 / 2}} \exp \left(-\frac{y^{2}}{4|1-t|}\right) \mathrm{d} y\right|=
\end{gathered}
$$




$$
=\frac{c}{1-t}\left|\int_{\mathbb{R}^{3}} \frac{\xi-\eta}{|\xi-\eta|^{3}} \exp \left(-\eta^{2}\right) \mathrm{d} \eta\right|=: \frac{A(\xi)}{1-t}
$$

where $\xi=x / 2 \sqrt{ }(1-t), \eta=y / 2 \sqrt{ }(1-t)$. Also

$$
\begin{gathered}
|\nabla(h * \Gamma(\cdot, t))(x)|^{2}=\sum_{i, j}\left|\partial_{i}\left(h^{j} * \Gamma(\cdot, t)\right)(x)\right|^{2}= \\
=\sum_{i, j}\left|\int_{\mathbb{R}^{3}} \frac{\delta_{i j}|x-y|^{2}-(x-y)_{i}(x-y)_{j}}{|x-y|^{3}} \frac{1}{(4 \pi|1-t|)^{3 / 2}} \exp \left(-\frac{y^{2}}{4|1-t|}\right) \mathrm{d} y\right|^{2}= \\
=\frac{c}{1-t} \sum_{i, j}\left|\int_{\mathbb{R}^{3}} \frac{\delta_{i j}|\xi-\eta|^{2}-(\xi-\eta)_{i}(\xi-\eta)_{j}}{|\xi-\eta|^{3}} \exp \left(-\eta^{2}\right) \mathrm{d} \eta\right|^{2}=: \\
=: \sum_{i, j} \frac{B_{i, j}^{2}(\xi)}{1-t}=: \frac{B(\xi)}{1-t} .
\end{gathered}
$$

Let $\xi$ be any vector in $\mathbb{R}^{3}$. By a rotation of our coordinate system we may assume that $\xi=\left(\xi_{1}, 0,0\right)$. Since $h$ commutes with rotations such a transformation does not alter $A$ or $B$. We estimate $B(\xi) \geqq B_{22}^{2}(\xi)$ and, using symmetry around the $x_{1}$-axis,

$$
\begin{gathered}
B_{22}(\xi)=c \int_{\mathbb{R}^{3}} \frac{(\xi-\eta)_{1}^{2}+(\xi-\eta)_{3}^{2}}{|\xi-\eta|^{3}} \exp \left(-\eta^{2}\right) \mathrm{d} \eta \geqq \\
\geqq c \int_{2 \eta_{2}{ }^{2} \leqq|\xi-\eta|^{2}} \frac{1}{|\xi-\eta|} \exp \left(-\eta^{2}\right) \mathrm{d} \eta \geqq c \int_{\mathbb{R}^{3}} \frac{1}{|\xi-\eta|} \exp \left(-\eta^{2}\right) \mathrm{d} \eta .
\end{gathered}
$$

Thus we obtain

$$
B(\zeta)>0, \quad B(\xi) \geqq c|\xi|^{-2}, \quad|\xi| \text { large } .
$$

Moreover, $B$ is a continuous function of $\xi$.

Similarly, $A$ is continuous as a function of $\xi$ and decays asymptotically at a rate

$$
A(\xi) \leqq c|\xi|^{-2}, \quad|\xi| \text { large } .
$$

Thus, the quotient $A / B$ is uniformly bounded, proving the lemma.

qed.

Let

$$
\begin{aligned}
& g(x, t, u, p)= \begin{cases}f(x, t, u, p), & t<1 \\
2 h(x) p^{2}, & t \geqq 1\end{cases} \\
& h(x, t, u, p)=\left\{\begin{array}{cc}
f(x, t, u, p), & t<1 \\
2 h(x) p^{2}, & t=1 \\
-f(x, 2-t, u, p), & t>1
\end{array}\right.
\end{aligned}
$$

Lemma 3. The functions $u, v$ are weak solutions in the sense of (4) of the parabolic systems

$$
\begin{array}{ll}
\partial_{t} u^{i}+L u^{i}=g^{i}(x, t, u, \nabla u), & 1 \leqq i \leqq 3 \\
\partial_{t} v^{i}+M v^{i}=h^{i}(x, t, v, \nabla v), & 1 \leqq i \leqq 3
\end{array}
$$


Proof. Clearly, if $\varphi$ is admissible in (4) and $\varphi$ vanishes in a neighborhood of $t=1$ then the analogues of (4) will be satisfied for $u$ and $v$, as follows from the derivation (11), (12) and Lemma 2 above. For an arbitrary testing function $\varphi$ let $\varphi_{1} \equiv \varphi(t \leqq 1)$, $\varphi_{1} \equiv 0(t>1)$ and let $\varphi_{2}=\varphi-\varphi_{1}$. Since by the remark after (10) $u$ and $v$ are continuous at $t=1$ in $L_{\mathrm{loc}}^{2}$ inserting $\varphi_{1}$ and $\varphi_{2}$ into the equations may be justified by an appropriate limiting process, yielding e.g. for the function $u$ :

$$
\begin{aligned}
& \int_{\Omega \times] 0,1[}\left[-u \partial_{t} \varphi_{1}+\alpha \nabla u \nabla \varphi_{1}\right] \mathrm{d} x \mathrm{~d} t=\int_{Q} g \varphi_{1} \mathrm{~d} x \mathrm{~d} t+\int_{\Omega \times\{0\}} u \varphi_{1} \mathrm{~d} x-\int_{\Omega \times\{1\}} u \varphi_{1} \mathrm{~d} x \\
& \int_{\Omega \times] 1, \infty[}\left[-u \partial_{t} \varphi_{2}+\alpha \nabla u \nabla \varphi_{2}\right] \mathrm{d} x \mathrm{~d} t=\int_{Q} g \varphi_{2} \mathrm{~d} x \mathrm{~d} t+\int_{\Omega \times\{1\}} u \varphi_{2} \mathrm{~d} x .
\end{aligned}
$$

Adding these identities and taking account of the continuity of $\varphi$ at $t=1$ the claim follows.

Similarly, the conclusion is obtained for $v$.

qed.

This concludes the construction. We remark that both $u$ and $v$ are solutions of uniformly parabolic diagonal systems with quadratic growth and smooth initial and boundary data but that develop a discontinuity in finite time. In the case of $u$ the singularity persists for all later times whereas in the case of $v$ the discontinuity immediately disappears again as $t$ is further increased.

\section{References}

[1] Frehse, J.: A discontinuous solution of a mildly nonlinear elliptic system, Math. Z. 134, 229-230 (1973).

[2] Giaquinta, $M$. and $M$. Struwe: An optimal regularity result for quasilinear parabolic systems, manusc. math. 36, $223-239$ (1981).

[3] Heinz, E.: On certain nonlinear elliptic differential equations and univalent mappings, J. Analyse math. 5, 197-272 (1956/57).

[4] Hildebrandt, $S$, and $K$.-O. Widman: Some regularity results for quasilinear elliptic systems of second order, Math. Z. 142, 67-86 (1975).

[5] Ladyshenskaya, O. A., V. A. Solonnikov and N. N. Ural'ceva: Linear and quasilinear equations of parabolic type, Transl. Math. Monographs 23, AMS, Providence, R. I. (1968).

[6] Sampson, J. H.: On the heat equation for harmonic maps, preprint.

[7] Struwe, M.: On the Hölder continuity of bounded weak solutions of quasilinear parabolic systems, Manusc. math. 35, 125-145 (1981).

Author's address: Mathematisches Institut der Universität Bonn, Beringstr. 6, 5300 Bonn 1, BRD. 